J. Korean Math. Soc. 49 (2012), No. 6, pp. 1123-1138

http://dx.doi.org/10.4134/JKMS.2012.49.6.1123

\title{
ANISOTROPIC QUASILINEAR ELLIPTIC EQUATIONS WITH VARIABLE EXPONENT
}

\author{
Mihai Mihăılescu and Denisa Stancu-Dumitru
}

\begin{abstract}
We study some anisotropic boundary value problems involving variable exponent growth conditions and we establish the existence and multiplicity of weak solutions by using as main argument critical point theory.
\end{abstract}

\section{Introduction}

Materials involving nonhomogenities are usually modelled by energetic functionals of the type

$$
\int|\nabla u(x)|^{p(x)} d x
$$

where $p(x)>1$ is a continuous function. Such kind of functionals are mentioned, for instance, in the work of Ruzicka [18] where they are used to model an electrorheological fluid. They correspond to the so called a $p(x)$-Laplace operator which is described by the formula

$$
\Delta_{p(x)} u=\operatorname{div}\left(|\nabla u|^{p(x)-2} \nabla u\right) .
$$

However, if we seek for the model of an inhomogeneous material which has a different behavior on each direction we note that the above energy is not adequate. In this new case an appropriate form for energetic functionals can be described by the formula

$$
\int \sum_{i}\left|\partial_{x_{i}} u\right|^{p_{i}(x)} d x
$$

where $p_{i}(x)>1$ are continuous functions. Functionals of type (2) correspond to a differential operator of the type

$$
\sum_{i} \partial_{x_{i}}\left(\left|\partial_{x_{i}} u\right|^{p_{i}(x)-2} \partial_{x_{i}} u\right)
$$

Received July 7, 2010

2010 Mathematics Subject Classification. 35J60, 35J62, 35J70.

Key words and phrases. variable exponent growth conditions, anisotropic equations, critical points.

(C)2012 The Korean Mathematical Society 
which appears also in a paper by Mihăilescu-Pucci-Rădulescu [11] and more recently in two papers by Mihăilescu-Moroşanu [9, 10]. Problems involving operators of type (3) will be called anisotropic partial differential equations with variable exponent. In the particular case when $p_{i}(x)=p(x)$ for each $i$ the differential operator (3) becomes $\sum_{i} \partial_{x_{i}}\left(\left|\partial_{x_{i}} u\right|^{p(x)-2} \partial_{x_{i}} u\right)$ and has similar properties with the $p(x)$-Laplace operator.

Motivated by the above discussion, we analyze in this paper the existence and multiplicity of solutions for a nonhomogeneous anisotropic problem of type

$$
\begin{cases}-\sum_{i=1}^{N} \partial_{x_{i}}\left(\left|\partial_{x_{i}} u\right|^{p_{i}(x)-2} \partial_{x_{i}} u\right)=f(x, u) & \text { for } x \in \Omega, \\ u=0 & \text { for } x \in \partial \Omega\end{cases}
$$

where $\Omega \subset \mathbb{R}^{N}(N \geq 3)$ is a bounded domain with smooth boundary and $p_{i}: \bar{\Omega} \rightarrow(1, \infty)$ are continuous functions for each $i \in\{1, \ldots, N\}$.

\section{A brief overview on variable exponent spaces}

Assume $\Omega \subset \mathbb{R}^{N}$ is an open domain.

Set

$$
C_{+}(\bar{\Omega})=\{h: h \in C(\bar{\Omega}), h(x)>1 \text { for all } x \in \bar{\Omega}\} .
$$

For any $p \in C_{+}(\bar{\Omega})$ we define

$$
p^{+}=\sup _{x \in \Omega} p(x) \quad \text { and } \quad p^{-}=\inf _{x \in \Omega} p(x) .
$$

For each $p \in C_{+}(\bar{\Omega})$, we recall the definition of the variable exponent Lebesgue space:

$$
\begin{gathered}
L^{p(\cdot)}(\Omega)=\{u: u \text { is a measurable real-valued function such that } \\
\left.\int_{\Omega}|u(x)|^{p(x)} d x<\infty\right\} .
\end{gathered}
$$

This space becomes a Banach space [7, Theorem 2.5] with respect to the Luxemburg norm, that is,

$$
|u|_{p(\cdot)}=\inf \left\{\mu>0: \int_{\Omega}\left|\frac{u(x)}{\mu}\right|^{p(x)} d x \leq 1\right\} .
$$

Moreover, $L^{p(\cdot)}(\Omega)$ is a reflexive space [7, Corollary 2.7] provided that $1<p^{-} \leq$ $p^{+}<\infty$. Furthermore, on such kind of spaces a Hölder type inequality is valid [7, Theorem 2.1]. More exactly, denoting by $L^{q(\cdot)}(\Omega)$ the conjugate space of $L^{p(\cdot)}(\Omega)$, where $\frac{1}{p(x)}+\frac{1}{q(x)}=1$ for any $x \in \bar{\Omega}$, for each $u \in L^{p(\cdot)}(\Omega)$ and each $v \in L^{q(\cdot)}(\Omega)$ the Hölder type inequality reads as follows

$$
\left|\int_{\Omega} u v d x\right| \leq\left(\frac{1}{p^{-}}+\frac{1}{q^{-}}\right)|u|_{p(\cdot)}|v|_{q(\cdot)} .
$$

An immediate consequence of Hölder's inequality is connected with some inclusions between various Lebesgue spaces involving variable exponent growth [7, Theorem 2.8]: if $0<|\Omega|<\infty$ and $p_{1}, p_{2}$ are variable exponents, such 
that $p_{1}(x) \leq p_{2}(x)$ almost everywhere in $\Omega$, then there exists the continuous embedding $L^{p_{2}(\cdot)}(\Omega) \hookrightarrow L^{p_{1}(\cdot)}(\Omega)$, whose norm does not exceed $|\Omega|+1$.

An important role in manipulating the generalized Lebesgue-Sobolev spaces is played by the modular of the $L^{p(\cdot)}(\Omega)$ space, which is the mapping $\rho_{p(\cdot)}$ : $L^{p(\cdot)}(\Omega) \rightarrow \mathbb{R}$ defined by

$$
\rho_{p(\cdot)}(u)=\int_{\Omega}|u|^{p(x)} d x,
$$

provided that $p^{+}<\infty$. Spaces with $p^{+}=\infty$ have been studied by Edmunds, Lang and Nekvinda [1].

We point out some relations which can be established between the Luxemburg norm and the modular. If $\left(u_{n}\right), u \in L^{p(\cdot)}(\Omega)$ and $p^{+}<\infty$, then the following relations hold true

$$
\begin{gathered}
|u|_{p(\cdot)}>1 \quad \Rightarrow \quad|u|_{p(\cdot)}^{p^{-}} \leq \rho_{p(\cdot)}(u) \leq|u|_{p(\cdot)}^{p^{+}}, \\
|u|_{p(\cdot)}<1 \quad \Rightarrow \quad|u|_{p(\cdot)}^{p^{+}} \leq \rho_{p(\cdot)}(u) \leq|u|_{p(\cdot)}^{p^{-}}, \\
\left|u_{n}-u\right|_{p(\cdot)} \rightarrow 0 \quad \Leftrightarrow \quad \rho_{p(\cdot)}\left(u_{n}-u\right) \rightarrow 0 .
\end{gathered}
$$

Next, we define the variable exponent Sobolev space $W_{0}^{1, p(\cdot)}(\Omega)$ as the closure of $C_{0}^{\infty}(\Omega)$ under the norm

$$
\|u\|=|\nabla u|_{p(\cdot)} \cdot
$$

The space $\left(W_{0}^{1, p(\cdot)}(\Omega),\|\cdot\|\right)$ is a separable and reflexive Banach space, provided that $1<p^{-} \leq p^{+}<\infty$. We recall that if $\Omega$ is a bounded, open domain in $\mathbb{R}^{N}$, $q \in C_{+}(\bar{\Omega})$ and $q(x)<p^{\star}(x)$ for all $x \in \bar{\Omega}$, then the embedding

$$
W_{0}^{1, p(\cdot)}(\Omega) \hookrightarrow L^{q(\cdot)}(\Omega)
$$

is compact and continuous, where $p^{\star}(x)=\frac{N p(x)}{N-p(x)}$ if $p(x)<N$ or $p^{\star}(x)=+\infty$ if $p(x) \geq N$. We refer to $[1,2,3,4,5,7,13]$ for further properties of variable exponent Lebesgue-Sobolev spaces.

Finally, we recall the definition and properties of the anisotropic variable exponent Sobolev spaces as they were introduced in [11]. With that end in view, we assume in the sequel that $\Omega$ is a bounded open domain in $\mathbb{R}^{N}$ and we denote by $\vec{p}(\cdot): \bar{\Omega} \rightarrow \mathbb{R}^{N}$ the vectorial function $\vec{p}(\cdot)=\left(p_{1}(\cdot), \ldots, p_{N}(\cdot)\right)$. We define $W_{0}^{1, \vec{p}(\cdot)}(\Omega)$, the anisotropic variable exponent Sobolev space, as the closure of $C_{0}^{\infty}(\Omega)$ with respect to the norm

$$
\|u\|_{\vec{p}(\cdot)}=\sum_{i=1}^{N}\left|\partial_{x_{i}} u\right|_{p_{i}(\cdot)} .
$$

In the case when $p_{i}(\cdot) \in C_{+}(\bar{\Omega})$ are constant functions for any $i \in\{1, \ldots, N\}$ the resulting anisotropic Sobolev space is denoted by $W_{0}^{1, \vec{p}}(\Omega)$, where $\vec{p}$ is the constant vector $\left(p_{1}, \ldots, p_{N}\right)$. The theory of this type of spaces was developed 
in $[6,14,16,17,20,21]$. It was argued in $[11]$ that $W_{0}^{1, \vec{p}(\cdot)}(\Omega)$ is a reflexive Banach space.

On the other hand, in order to facilitate the manipulation of the space $W_{0}^{1, \vec{p}(\cdot)}(\Omega)$ we introduce $\vec{P}_{+}, \vec{P}_{-}$in $\mathbb{R}^{N}$ as

$$
\vec{P}_{+}=\left(p_{1}^{+}, \ldots, p_{N}^{+}\right), \quad \vec{P}_{-}=\left(p_{1}^{-}, \ldots, p_{N}^{-}\right),
$$

and $P_{+}^{+}, P_{-}^{+}, P_{-}^{-} \in \mathbb{R}^{+}$as

$$
P_{+}^{+}=\max \left\{p_{1}^{+}, \ldots, p_{N}^{+}\right\}, \quad P_{-}^{+}=\max \left\{p_{1}^{-}, \ldots, p_{N}^{-}\right\}, \quad P_{-}^{-}=\min \left\{p_{1}^{-}, \ldots, p_{N}^{-}\right\} .
$$

Throughout this paper we assume that

$$
\sum_{i=1}^{N} \frac{1}{p_{i}^{-}}>1
$$

and define $P_{-}^{\star} \in \mathbb{R}^{+}$and $P_{-, \infty} \in \mathbb{R}^{+}$by

$$
P_{-}^{\star}=\frac{N}{\sum_{i=1}^{N} \frac{1}{p_{i}^{-}}-1}, \quad P_{-, \infty}=\max \left\{P_{-}^{+}, P_{-}^{\star}\right\} .
$$

Finally, we recall a result regarding the compact embedding between $W_{0}^{1, \vec{p}(\cdot)}(\Omega)$ and variable exponent Lebesgue spaces (see, [11, Theorem 1]):

Theorem 1. Assume that $\Omega \subset \mathbb{R}^{N}(N \geq 3)$ is a bounded domain with smooth boundary. Assume relation (9) is fulfilled. For any $q \in C(\bar{\Omega})$ verifying

$$
1<q(x)<P_{-, \infty} \text { for all } x \in \bar{\Omega},
$$

the embedding

$$
W_{0}^{1, \vec{p}(\cdot)}(\Omega) \hookrightarrow L^{q(\cdot)}(\Omega)
$$

is continuous and compact.

\section{The main results}

In this paper we study problem (4) in the particular cases

$$
f(x, t)= \pm\left(-\lambda|t|^{m(x)-2} t+|t|^{q(x)-2} t\right)
$$

where $m: \bar{\Omega} \rightarrow \mathbb{R}, q: \bar{\Omega} \rightarrow \mathbb{R}$ are continuous functions such that

$$
\begin{aligned}
& m(x)=\max _{i \in\{1, \ldots, N\}}\left\{p_{i}(x)\right\} \text { for any } x \in \bar{\Omega}, \\
& 1<m(x)<q(x)<P_{-, \infty} \text { for any } x \in \bar{\Omega}
\end{aligned}
$$

and $\lambda>0$.

Remark. Condition (11) implies $m^{+}=P_{+}^{+}$.

First, we consider the following problem

$$
\begin{cases}-\sum_{i=1}^{N} \partial_{x_{i}}\left(\left|\partial_{x_{i}} u\right|^{p_{i}(x)-2} \partial_{x_{i}} u\right)=-\lambda|u|^{m(x)-2} u+|u|^{q(x)-2} u & \text { for } x \in \Omega, \\ u=0 & \text { for } x \in \partial \Omega .\end{cases}
$$


We seek solutions for problem (13) belonging to the space $W_{0}^{1, \vec{p}(\cdot)}(\Omega)$ in the sense below.

Definition 1. We say that $u \in W_{0}^{1, \vec{p}(\cdot)}(\Omega)$ is a weak solution for problem (13) if

$$
\int_{\Omega}\left\{\sum_{i=1}^{N}\left(\left|\partial_{x_{i}} u\right|^{p_{i}(x)-2} \partial_{x_{i}} u \partial_{x_{i}} v\right)+\lambda|u|^{m(x)-2} u v-|u|^{q(x)-2} u v\right\} d x=0
$$

for all $v \in W_{0}^{1, \vec{p}(\cdot)}(\Omega)$.

We will prove:

Theorem 2. For every $\lambda>0$ problem (13) has infinitely many weak solutions provided $2 \leq P_{-}^{-}, P_{+}^{+}<q^{-}$and $q^{+}<P_{-, \infty}$.

Next, we deal with the problem

$$
\begin{cases}-\sum_{i=1}^{N} \partial_{x_{i}}\left(\left|\partial_{x_{i}} u\right|^{p_{i}(x)-2} \partial_{x_{i}} u\right)=\lambda|u|^{m(x)-2} u-|u|^{q(x)-2} u & \text { for } x \in \Omega \\ u=0 & \text { for } x \in \partial \Omega .\end{cases}
$$

We seek solutions for problem (14) belonging to the space $W_{0}^{1, \vec{p}(\cdot)}(\Omega)$ in the sense below.

Definition 2. We say that $u \in W_{0}^{1, \vec{p}(\cdot)}(\Omega)$ is a weak solution for problem (14) if

$$
\int_{\Omega}\left\{\sum_{i=1}^{N}\left(\left|\partial_{x_{i}} u\right|^{p_{i}(x)-2} \partial_{x_{i}} u \partial_{x_{i}} v\right)-\lambda|u|^{m(x)-2} u v+|u|^{q(x)-2} u v\right\} d x=0
$$

for all $v \in W_{0}^{1, \vec{p}(\cdot)}(\Omega)$.

Regarding problem (14) we prove the following result:

Theorem 3. There exists $\lambda^{\star}>0$ such that for any $\lambda \geq \lambda^{\star}$ problem (14) has a nontrivial weak solution provided $2 \leq P_{-}^{-}, P_{+}^{+}<q^{-}$and $q^{+}<P_{-, \infty}$.

Remark. We point out the fact that similar results as the one of Theorems 2 and 3 were obtained by Mihăilescu [8], in the case when in the left hand side of equations (13) and (14) we replace the anisotropic operator

$$
\sum_{i=1}^{N} \partial_{x_{i}}\left(\left|\partial_{x_{i}} u\right|^{p_{i}(x)-2} \partial_{x_{i}} u\right)
$$

by an isotropic one of the type $\operatorname{div}\left(\left(|\nabla u|^{p_{1}(x)-2}+|\nabla u|^{p_{2}(x)-2}\right) \nabla u\right)$, where $p_{1}(x)$ and $p_{2}(x)$ are two continuous functions. Our results represent a natural generalization of the one in [8] in the anisotropic case. 


\section{Proof of Theorem 2}

We will use critical point theory to prove Theorem 2. More exactly, we will associate to problem (13) an energetic functional for which the critical points correspond to the weak solutions of the equation. The main tool is a $\mathbb{Z}_{2}$-symmetric version (for even functionals) of the Mountain Pass Theorem (see $[15$, Theorem 9.12]):

Mountain Pass Theorem. Let $X$ be an infinite dimensional real Banach space and let $I \in C^{1}(X, \mathbb{R})$ be even, satisfying the Palais-Smale condition (that is, any sequence $\left\{x_{n}\right\} \subset X$ such that $\left\{I\left(x_{n}\right)\right\}$ is bounded and $I^{\prime}\left(x_{n}\right) \rightarrow 0$ in $X^{\star}$ has a convergent subsequence) and $I(0)=0$. Suppose that

(I1) there exist two constants $\rho, a>0$ such that $I(x) \geq a$ if $\|x\|_{X}=\rho$,

(I2) for each finite dimensional subspace $X_{1} \subset X$, the set $\left\{x \in X_{1}: I(x) \geq\right.$ $0\}$ is bounded.

Then I has an unbounded sequence of critical values.

Let $\lambda>0$ be arbitrary but fixed. Define the energy functional $I_{\lambda}: W_{0}^{1, \vec{p}(\cdot)}(\Omega)$ $\rightarrow \mathbb{R}$, corresponding to problem (13), by

$$
I_{\lambda}(u)=\int_{\Omega}\left\{\sum_{i=1}^{N} \frac{\left|\partial_{x_{i}} u\right|^{p_{i}(x)}}{p_{i}(x)}+\lambda \frac{|u|^{m(x)}}{m(x)}-\frac{|u|^{q(x)}}{q(x)}\right\} d x .
$$

Standard arguments assure that $I_{\lambda} \in C^{1}\left(W_{0}^{1, \vec{p}(\cdot)}(\Omega), \mathbb{R}\right)$ and the Fréchet derivative is given by

$$
\left\langle I_{\lambda}^{\prime}(u), v\right\rangle=\int_{\Omega}\left\{\sum_{i=1}^{N}\left|\partial_{x_{i}} u\right|^{p_{i}(x)-2} \partial_{x_{i}} u \partial_{x_{i}} v+\lambda|u|^{m(x)-2} u v-|u|^{q(x)-2} u v\right\} d x
$$

for all $u, v \in W_{0}^{1, \vec{p}(\cdot)}(\Omega)$. Obviously, the weak solutions of problem (13) coincide with the critical points of $I_{\lambda}$.

Our goal is to show that the Mountain Pass Theorem can be applied in this case. In order to do that we start by establishing some auxiliary results.

Lemma 1. If $\left\{u_{n}\right\} \subset W_{0}^{1, \vec{p}(\cdot)}(\Omega)$ is a sequence which satisfies the conditions

$$
\begin{gathered}
\left|I_{\lambda}(u)\right|<K, \\
I_{\lambda}^{\prime}\left(u_{n}\right) \rightarrow 0 \text { as } n \rightarrow \infty,
\end{gathered}
$$

where $K$ is a positive constant, then $\left\{u_{n}\right\}$ has a convergent subsequence.

Proof. First, we show that $\left\{u_{n}\right\}$ is bounded in $W_{0}^{1, \vec{p}(\cdot)}(\Omega)$. In order to do that, we assume by contradiction that passing eventually to a subsequence, still denoted by $\left\{u_{n}\right\}$, we have $\left\|u_{n}\right\|_{\vec{p}(\cdot)} \rightarrow \infty$ as $n \rightarrow \infty$. Clearly, we may assume that $\left\|u_{n}\right\|_{\vec{p}(\cdot)}>1$ for any integer $n$. 
Condition (18) implies that for $n$ large enough we have

$$
\left\|I_{\lambda}^{\prime}\left(u_{n}\right)\right\| \leq 1 .
$$

On the other hand, for each fixed $n$, the application

$$
W_{0}^{1, \vec{p}(\cdot)}(\Omega) \ni v \longmapsto\left\langle I_{\lambda}^{\prime}\left(u_{n}\right), v\right\rangle \in \mathbb{R}
$$

is linear and continuous. Combining the above two relations, we obtain that

$$
\left|\left\langle I_{\lambda}^{\prime}\left(u_{n}\right), v\right\rangle\right| \leq\left\|I_{\lambda}^{\prime}\left(u_{n}\right)\right\| \cdot\|v\|_{\vec{p}(\cdot)} \leq\|v\|_{\vec{p}(\cdot)}, \quad \forall v \in W_{0}^{1, \vec{p}(\cdot)}(\Omega),
$$

for $n$ large enough. Setting $v=u_{n}$, we deduce that

$$
\begin{aligned}
-\left\|u_{n}\right\|_{\vec{p}(\cdot)} & \leq \int_{\Omega} \sum_{i=1}^{N}\left|\partial_{x_{i}} u_{n}\right|^{p_{i}(x)} d x+\lambda \int_{\Omega}\left|u_{n}\right|^{m(x)} d x-\int_{\Omega}\left|u_{n}\right|^{q(x)} d x \\
& \leq\left\|u_{n}\right\|_{\vec{p}(\cdot)}
\end{aligned}
$$

for $n$ large enough.

Thus, the above information yields

(19) $-\left\|u_{n}\right\|_{\vec{p}(\cdot)}-\int_{\Omega} \sum_{i=1}^{N}\left|\partial_{x_{i}} u_{n}\right|^{p_{i}(x)} d x-\lambda \int_{\Omega}\left|u_{n}\right|^{m(x)} d x \leq-\int_{\Omega}\left|u_{n}\right|^{q(x)} d x$ for all $n$ large.

Provided that $\left\|u_{n}\right\|_{\vec{p}(\cdot)}>1$, by relations (17), (19) and (6), and the fact that $m^{+}=P_{+}^{+}$we get

$$
\begin{aligned}
K> & I_{\lambda}\left(u_{n}\right) \\
\geq & \frac{1}{P_{+}^{+}} \int_{\Omega} \sum_{i=1}^{N}\left|\partial_{x_{i}} u_{n}\right|^{p_{i}(x)} d x+\frac{\lambda}{m^{+}} \int_{\Omega}\left|u_{n}\right|^{m(x)} d x-\frac{1}{q^{-}} \int_{\Omega}\left|u_{n}\right|^{q(x)} d x \\
\geq & \left(\frac{1}{P_{+}^{+}}-\frac{1}{q^{-}}\right) \int_{\Omega} \sum_{i=1}^{N}\left|\partial_{x_{i}} u_{n}\right|^{p_{i}(x)} d x+\lambda\left(\frac{1}{m^{+}}-\frac{1}{q^{-}}\right) \int_{\Omega}\left|u_{n}\right|^{m(x)} d x \\
& -\frac{1}{q^{-}}\left\|u_{n}\right\|_{\vec{p}(\cdot)} \\
\geq & \left(\frac{1}{P_{+}^{+}}-\frac{1}{q^{-}}\right) \int_{\Omega} \sum_{i=1}^{N}\left|\partial_{x_{i}} u_{n}\right|^{p_{i}(x)} d x-\frac{1}{q^{-}}\left\|u_{n}\right\|_{\vec{p}(\cdot)} .
\end{aligned}
$$

For each $n$ and $i \in\{1, \ldots, N\}$ we define

$$
\xi_{n, i}= \begin{cases}P_{+}^{+}, & \text {if }\left|\partial_{x_{i}} u_{n}\right|_{p_{i}(\cdot)}<1 \\ P_{-}^{-}, & \text {if }\left|\partial_{x_{i}} u_{n}\right|_{p_{i}(\cdot)}>1\end{cases}
$$

We have

$$
K>I_{\lambda}\left(u_{n}\right) \geq\left(\frac{1}{P_{+}^{+}}-\frac{1}{q^{-}}\right) \int_{\Omega} \sum_{i=1}^{N}\left|\partial_{x_{i}} u_{n}\right|^{p_{i}(x)} d x-\frac{1}{q^{-}}\left\|u_{n}\right\|_{\vec{p}(\cdot)}
$$




$$
\begin{aligned}
\geq & \left(\frac{1}{P_{+}^{+}}-\frac{1}{q^{-}}\right) \sum_{i=1}^{N}\left|\partial_{x_{i}} u_{n}\right|_{p_{i}(\cdot)}^{\xi_{n, i}}-\frac{1}{q^{-}}\left\|u_{n}\right\|_{\vec{p}(\cdot)} \\
\geq & \left(\frac{1}{P_{+}^{+}}-\frac{1}{q^{-}}\right) \sum_{i=1}^{N}\left|\partial_{x_{i}} u_{n}\right|_{p_{i}(\cdot)}^{P_{-}^{-}} \\
& -\left(\frac{1}{P_{+}^{+}}-\frac{1}{q^{-}}\right) \sum_{\left\{i: \xi_{n, i}=P_{+}^{+}\right\}}\left(\left|\partial_{x_{i}} u_{n}\right|_{p_{i}(\cdot)}^{P^{-}}-\left|\partial_{x_{i}} u_{n}\right|_{p_{i}(\cdot)}^{P_{+}^{+}}\right)-\frac{1}{q^{-}}\left\|u_{n}\right\|_{\vec{p}(\cdot)} \\
\geq & \left(\frac{1}{P_{+}^{+}}-\frac{1}{q^{-}}\right) \frac{\left\|u_{n}\right\|_{\vec{p}(\cdot)}^{P^{-}}}{N^{P_{-}^{-}-1}}-N\left(\frac{1}{P_{+}^{+}}-\frac{1}{q^{-}}\right)-\frac{1}{q^{-}}\left\|u_{n}\right\|_{\vec{p}(\cdot) \cdot}
\end{aligned}
$$

Passing to the limit as $n \rightarrow \infty$, we obtain a contradiction. It follows that $\left\{u_{n}\right\}$ is bounded in $W_{0}^{1, \vec{p}(\cdot)}(\Omega)$.

Since $\left\{u_{n}\right\}$ is bounded in $W_{0}^{1, \vec{p}(\cdot)}(\Omega)$ and the space $W_{0}^{1, \vec{p}(\cdot)}(\Omega)$ is reflexive, we deduce that there exist a subsequence, still denoted by $\left\{u_{n}\right\}$, and $u$ in $W_{0}^{1, \vec{p}(\cdot)}(\Omega)$ such that $\left\{u_{n}\right\}$ converges weakly to $u$ in $W_{0}^{1, \vec{p}(\cdot)}(\Omega)$. Theorem 1 and conditions (11) and (12) imply that $W_{0}^{1, \vec{p}(\cdot)}(\Omega)$ is compactly embedded into $L^{m(\cdot)}(\Omega)$ and $L^{q(\cdot)}(\Omega)$. Consequently, $\left\{u_{n}\right\}$ converges strongly to $u$ in $L^{m(\cdot)}(\Omega)$ and $L^{q(\cdot)}(\Omega)$.

These facts and condition (18) show that

$$
\left\langle I_{\lambda}^{\prime}\left(u_{n}\right)-I_{\lambda}^{\prime}(u), u_{n}-u\right\rangle \rightarrow 0 \text { as } n \rightarrow \infty
$$

We get

$$
\begin{aligned}
& \int_{\Omega} \sum_{i=1}^{N}\left(\left|\partial_{x_{i}} u_{n}\right|^{p_{i}(x)-2} \cdot \partial_{x_{i}} u_{n}-\left|\partial_{x_{i}} u\right|^{p_{i}(x)-2} \cdot \partial_{x_{i}} u\right) \cdot\left(\partial_{x_{i}} u_{n}-\partial_{x_{i}} u\right) d x \\
= & \left\langle I_{\lambda}^{\prime}\left(u_{n}\right)-I_{\lambda}^{\prime}(u), u_{n}-u\right\rangle-\lambda \int_{\Omega}\left(\left|u_{n}\right|^{m(x)-2} u_{n}-|u|^{m(x)-2} u\right) \cdot\left(u_{n}-u\right) d x \\
& +\int_{\Omega}\left(\left|u_{n}\right|^{q(x)-2} u_{n}-|u|^{q(x)-2} u\right) \cdot\left(u_{n}-u\right) d x .
\end{aligned}
$$

Using the fact that $\left\{u_{n}\right\}$ converges strongly to $u$ in $L^{q(\cdot)}(\Omega)$ and inequality (5) we get

$$
\begin{aligned}
& \left|\int_{\Omega}\left(\left|u_{n}\right|^{q(x)-2} u_{n}-|u|^{q(x)-2} u\right)\left(u_{n}-u\right) d x\right| \\
\leq & \left.\left|\int_{\Omega}\right| u_{n}\right|^{q(x)-2} u_{n}\left(u_{n}-u\right) d x|+| \int_{\Omega}|u|^{q(x)-2} u\left(u_{n}-u\right) d x \mid \\
\leq & \left.\left.M_{1}|| u_{n}\right|^{q(x)-1}\right|_{\frac{q(\cdot)}{q(\cdot)-1}} \cdot\left|u_{n}-u\right|_{q(\cdot)}+\left.\left.M_{2}|| u\right|^{q(x)-1}\right|_{\frac{q(\cdot)}{q(\cdot)-1}} \cdot\left|u_{n}-u\right|_{q(\cdot),},
\end{aligned}
$$

where $M_{1}, M_{2}$ are two positive constants. 
Since $\left|u_{n}-u\right|_{q(\cdot)} \rightarrow 0$ as $n \rightarrow \infty$, we deduce that

$$
\lim _{n \rightarrow \infty} \int_{\Omega}\left(\left|u_{n}\right|^{q(x)-2} u_{n}-|u|^{q(x)-2} u\right)\left(u_{n}-u\right) d x=0 .
$$

Similar arguments as the one used in the proof of relation (21) show that

$$
\lim _{n \rightarrow \infty} \int_{\Omega}\left(\left|u_{n}\right|^{m(x)-2} u_{n}-|u|^{m(x)-2} u\right)\left(u_{n}-u\right) d x=0 .
$$

By relations (20), (21) and (22) we have

$$
\lim _{n \rightarrow \infty} \int_{\Omega} \sum_{i=1}^{N}\left(\left|\partial_{x_{i}} u_{n}\right|^{p_{i}(x)-2} \partial_{x_{i}} u_{n}-\left|\partial_{x_{i}} u\right|^{p_{i}(x)-2} \partial_{x_{i}} u\right)\left(\partial_{x_{i}} u_{n}-\partial_{x_{i}} u\right) d x=0 .
$$

It is known that

$$
\left(|\zeta|^{t-2} \zeta-|\vartheta|^{t-2} \vartheta\right)(\zeta-\vartheta) \geq 2^{-t}|\zeta-\vartheta|^{t}, \quad \forall t \geq 2, \quad \forall \zeta, \vartheta \in \mathbb{R}^{N}
$$

Relations (23) and (24) yield that actually $\left\{u_{n}\right\}$ converges strongly to $u$ in $W_{0}^{1, \vec{p}(\cdot)}(\Omega)$. The proof of Lemma 1 is complete.

Lemma 2. There exist $\rho>0$ and $a>0$ such that

$$
I_{\lambda}(u) \geq a>0, \quad \forall u \in W_{0}^{1, \vec{p}(\cdot)}(\Omega) \text { with }\|u\|_{\vec{p}(\cdot)}=\rho .
$$

Proof. By condition (12) we have $1<q^{-} \leq q^{+}<P_{-, \infty}$, for all $x \in \bar{\Omega}$ and using Theorem 1 we get that $W_{0}^{1, \vec{p}(\cdot)}(\Omega)$ is compactly embedded in $L^{q^{-}}(\Omega)$ and $L^{q^{+}}(\Omega)$.

The fact that $W_{0}^{1, \vec{p}(\cdot)}(\Omega)$ is compactly embedded in $L^{q^{-}}(\Omega)$ assures that there exists a positive constant $C_{1}$ such that

$$
|u|_{q^{-}} \leq C_{1} \cdot\|u\|_{\vec{p}(\cdot)}, \quad \forall u \in W_{0}^{1, \vec{p}(\cdot)}(\Omega) .
$$

Similarly, $W_{0}^{1, \vec{p}(\cdot)}(\Omega)$ is compactly embedded in $L^{q^{+}}(\Omega)$ and this guarantees that there exists a positive constant $C_{2}$ such that

$$
|u|_{q^{+}} \leq C_{2} \cdot\|u\|_{\vec{p}(\cdot)}, \quad \forall u \in W_{0}^{1, \vec{p}(\cdot)}(\Omega) .
$$

On the other hand, we have

$$
|u(x)|^{q(x)} \leq|u(x)|^{q^{-}}+|u(x)|^{q^{+}} \text {for all } x \in \bar{\Omega} .
$$

Using relation $(27)$ we deduce that

$$
I_{\lambda}(u) \geq \frac{1}{P_{+}^{+}} \int_{\Omega} \sum_{i=1}^{N}\left|\partial_{x_{i}} u\right|^{p_{i}(x)} d x-\frac{1}{q^{-}}\left(\int_{\Omega}|u|^{q^{-}} d x+\int_{\Omega}|u|^{q^{+}} d x\right) .
$$

Next, we focus our attention on the case when $u \in W_{0}^{1, \vec{p}(\cdot)}(\Omega)$ with $\|u\|_{\vec{p}(\cdot)}$ $<1$. For such an element $u$, we have $\left|\partial_{x_{i}} u\right|_{p_{i}(\cdot)}<1$ for any $i \in\{1, \ldots, N\}$, 
and, by relation $(7)$ we obtain

$$
\begin{aligned}
\frac{\|u\|_{\vec{p}(\cdot)}^{P_{+}^{+}}}{N^{P_{+}^{+}-1}} & =N\left(\frac{\sum_{i=1}^{N}\left|\partial_{x_{i}} u\right|_{p_{i}(\cdot)}}{N}\right)^{P_{+}^{+}} \\
& \leq \sum_{i=1}^{N}\left|\partial_{x_{i}} u\right|_{p_{i}(\cdot)}^{P_{+}^{+}} \leq \sum_{i=1}^{N}\left|\partial_{x_{i}} u\right|_{p_{i}(\cdot)}^{p_{i}^{+}} \leq \sum_{i=1}^{N} \int_{\Omega}\left|\partial_{x_{i}} u\right|^{p_{i}(x)} d x .
\end{aligned}
$$

Thus, relations (25), (26), (28) and (29) imply

$$
\begin{aligned}
I_{\lambda}(u) & \geq \frac{1}{P_{+}^{+}} \frac{\|u\|_{\vec{p}(\cdot)}^{P_{+}^{+}}}{N^{P_{+}^{+}-1}}-C_{3}\|u\|_{\vec{p}(\cdot)}^{q^{-}}-C_{4}\|u\|_{\frac{\vec{p}(\cdot)}{q^{+}}} \\
& =\left(B_{1}-B_{2}\|u\|_{\vec{p}(\cdot)}^{q^{-}-P_{+}^{+}}-B_{3}\|u\|_{\vec{p}(\cdot)}^{q^{+}-P_{+}^{+}}\right)\|u\|_{\vec{p}(\cdot)}^{P_{+}^{+}}
\end{aligned}
$$

for any $u \in W_{0}^{1, \vec{p}(\cdot)}(\Omega)$ with $\|u\|_{\vec{p}(\cdot)}<1$, where $C_{3}, C_{4}, B_{1}, B_{2}$ and $B_{3}$ are positive constants.

Since the function $g:[0,1] \rightarrow \mathbb{R}$ defined by

$$
g(t)=B_{1}-B_{2} \cdot t^{q^{-}-P_{+}^{+}}-B_{3} \cdot t^{q^{+}-P_{+}^{+}}
$$

is positive in a neighborhood of the origin, the conclusion of the lemma follows at once.

Lemma 3. If $S$ is a finite dimensional subspace of $W_{0}^{1, \vec{p}(\cdot)}(\Omega)$, the set $M=$ $\left\{u \in S: I_{\lambda}(u) \geq 0\right\}$ is bounded in $W_{0}^{1, \vec{p}(\cdot)}(\Omega)$.

Proof. First, we establish that

$$
\sum_{i=1}^{N} \int_{\Omega} \frac{\left|\partial_{x_{i}} u\right|^{p_{i}(x)}}{p_{i}(x)} d x \leq A_{1}\left(\|u\|_{\vec{p}(\cdot)}^{P_{+}^{+}}+\|u\|_{\vec{p}(\cdot)}^{P_{-}^{-}}\right) \text {for all } u \in W_{0}^{1, \vec{p}(\cdot)}(\Omega),
$$

where $A_{1}=\frac{2 N}{P_{-}^{-}}$is a positive constant.

Indeed, using relations (6) and (7) we get

$$
\begin{aligned}
\sum_{i=1}^{N} \int_{\Omega} \frac{\left|\partial_{x_{i}} u\right|^{p_{i}(x)}}{p_{i}(x)} d x & \leq \sum_{i=1}^{N} \frac{1}{p_{i}^{-}}\left(\left|\partial_{x_{i}} u\right|_{p_{i}(\cdot)}^{p_{i}^{-}}+\left|\partial_{x_{i}} u\right|_{p_{i}(\cdot)}^{p_{i}^{+}}\right) \\
& \leq \frac{1}{P_{-}^{-}} \sum_{i=1}^{N}\left(\left|\partial_{x_{i}} u\right|_{p_{i}(\cdot)}^{p_{i}^{-}}+\left|\partial_{x_{i}} u\right|_{p_{i}(\cdot)}^{p_{i}^{+}}\right)
\end{aligned}
$$

for all $u \in W_{0}^{1, \vec{p}(\cdot)}(\Omega)$.

On the other hand, for every $i \in\{1, \ldots, N\}$ and for all $u \in W_{0}^{1, \vec{p}(\cdot)}(\Omega)$ we infer that

$$
\left|\partial_{x_{i}} u\right|_{p_{i}(\cdot)}^{p_{i}^{-}} \leq\|u\|_{\vec{p}(\cdot)}^{p_{i}^{-}} \leq\|u\|_{\vec{p}(\cdot)}^{P_{-}^{-}}+\|u\|_{\vec{p}(\cdot)}^{P_{+}^{+}}
$$


and

$$
\left|\partial_{x_{i}} u\right|_{p_{i}(\cdot)}^{p_{i}^{+}} \leq\|u\|_{\vec{p}(\cdot)}^{p_{i}^{+}} \leq\|u\|_{\vec{p}(\cdot)}^{P_{-}^{-}}+\|u\|_{\vec{p}(\cdot)}^{P_{+}^{+}} .
$$

The above three inequalities yield

$$
\sum_{i=1}^{N} \int_{\Omega} \frac{\left|\partial_{x_{i}} u\right|^{p_{i}(x)}}{p_{i}(x)} d x \leq \frac{2 N}{P_{-}^{-}}\left(\|u\|_{\vec{p}(\cdot)}^{P_{-}^{-}}+\|u\|_{\vec{p}(\cdot)}^{P_{+}^{+}}\right) .
$$

Thus, we conclude that inequality (30) holds true.

By relations (6) and (7), we arrive at

$$
\int_{\Omega}|u|^{m(x)} d x \leq|u|_{m(\cdot)}^{m^{-}}+|u|_{m(\cdot)}^{m^{+}} \text {for all } u \in W_{0}^{1, \vec{p}(\cdot)}(\Omega) .
$$

The fact that $W_{0}^{1, \vec{p}(\cdot)}(\Omega)$ is continuously embedded in $L^{m(\cdot)}(\Omega)$ guarantees that there exists a positive constant $H$ such that

$$
|u|_{m(\cdot)} \leq H\|u\|_{\vec{p}(\cdot)} \text { for all } u \in W_{0}^{1, \vec{p}(\cdot)}(\Omega)
$$

Combining inequalities (32) and (33) we obtain that, for each $\lambda>0$, there exists a positive constant $A_{2}(\lambda)$ such that

(34) $\lambda \cdot \int_{\Omega} \frac{|u|^{m(x)}}{m(x)} d x \leq A_{2}(\lambda)\left(\|u\|_{\vec{p}(\cdot)}^{m^{+}}+\|u\|_{\vec{p}(\cdot)}^{m^{-}}\right)$for all $u \in W_{0}^{1, \vec{p}(\cdot)}(\Omega)$.

Relations (30) and (34) imply

$I_{\lambda}(u) \leq A_{1}\left(\|u\|_{\vec{p}(\cdot)}^{P_{+}^{+}}+\|u\|_{\vec{p}(\cdot)}^{P_{-}^{-}}\right)+A_{2}(\lambda)\left(\|u\|_{\vec{p}(\cdot)}^{m^{+}}+\|u\|_{\vec{p}(\cdot)}^{m^{-}}\right)-\frac{1}{q^{+}} \int_{\Omega}|u|^{q(x)} d x$ for all $u \in W_{0}^{1, \vec{p}(\cdot)}(\Omega)$.

Let $u \in W_{0}^{1, \vec{p}(\cdot)}(\Omega)$ be arbitrary but fixed. We denote by

$$
\Omega_{<}:=\{x \in \Omega:|u(x)|<1\} \text { and } \Omega_{\geq}:=\Omega \backslash \Omega_{<} .
$$

Thus, we obtain

$$
\begin{aligned}
I_{\lambda}(u) \leq & A_{1}\left(\|u\|_{\vec{p}(\cdot)}^{P_{+}^{+}}+\|u\|_{\vec{p}(\cdot)}^{P_{-}^{-}}\right)+A_{2}(\lambda)\left(\|u\|_{\vec{p}(\cdot)}^{\frac{m^{+}}{p^{\prime}}}+\|u\| \|_{\vec{p}(\cdot)}^{m^{-}}\right) \\
& -\frac{1}{q^{+}} \int_{\Omega}|u|^{q(x)} d x \\
\leq & A_{1}\left(\|u\|_{\vec{p}(\cdot)}^{P_{+}^{+}}+\|u\|_{\vec{p}(\cdot)}^{P_{-}^{-}}\right)+A_{2}(\lambda)\left(\|u\|_{\vec{p}(\cdot)}^{m^{+}}+\|u\| \frac{m_{\vec{p}}^{-}(\cdot)}{p^{-}}\right) \\
& -\frac{1}{q^{+}} \int_{\Omega_{\geq}}|u|^{q(x)} d x \\
\leq & A_{1}\left(\|u\|_{\vec{p}(\cdot)}^{P_{+}^{+}}+\|u\|_{\vec{p}(\cdot)}^{P_{-}^{-}}\right)+A_{2}(\lambda)\left(\|u\|_{\vec{p}(\cdot)}^{m^{+}}+\|u\|_{\vec{p}(\cdot)}^{m^{-}}\right) \\
& -\frac{1}{q^{+}} \int_{\Omega_{\geq}}|u|^{q^{-}} d x
\end{aligned}
$$




$$
\begin{aligned}
\leq & A_{1}\left(\|u\|_{\vec{p}(\cdot)}^{P_{+}^{+}}+\|u\|_{\vec{p}(\cdot)}^{P_{-}^{-}}\right)+A_{2}(\lambda)\left(\|u\|_{\vec{p}(\cdot)}^{m^{+}}+\|u\|_{\vec{p}(\cdot)}^{m^{-}}\right) \\
& -\frac{1}{q^{+}} \int_{\Omega}|u|^{q^{-}} d x+\frac{1}{q^{+}} \int_{\Omega_{<}}|u|^{q^{-}} d x .
\end{aligned}
$$

But there exists a positive constant $A_{3}$ such that

$$
\frac{1}{q^{+}} \int_{\Omega_{<}}|u|^{q^{-}} d x \leq A_{3}, \quad \forall u \in W_{0}^{1, \vec{p}(\cdot)}(\Omega) .
$$

Then, we have

$$
\begin{aligned}
I_{\lambda}(u) \leq & A_{1}\left(\|u\|_{\vec{p}(\cdot)}^{P_{+}^{+}}+\|u\|_{\vec{p}(\cdot)}^{P_{-}^{-}}\right)+A_{2}(\lambda)\left(\|u\|_{\vec{p}(\cdot)}^{m^{+}}+\|u\|_{\vec{p}(\cdot)}^{m^{-}}\right) \\
& -\frac{1}{q^{+}} \int_{\Omega}|u|^{q^{-}} d x+A_{3}
\end{aligned}
$$

for all $u \in W_{0}^{1, \vec{p}(\cdot)}(\Omega)$.

Define the functional $|\cdot|_{q^{-}}: W_{0}^{1, \vec{p}(\cdot)}(\Omega) \rightarrow \mathbb{R}$ by

$$
|u|_{q^{-}}:=\left(\int_{\Omega}|u|^{q^{-}} d x\right)^{1 / q^{-}} .
$$

The functional $|\cdot|_{q^{-}}$is a norm on $W_{0}^{1, \vec{p}(\cdot)}(\Omega)$. On the finite dimensional subspace $S$, the norms $|\cdot|_{q^{-}}$and $\|\cdot\|_{\vec{p}(\cdot)}$ are equivalent, so there exists a positive constant $A=A(S)$ such that

$$
\|u\|_{\vec{p}(\cdot)} \leq A \cdot|u|_{q^{-}}, \quad \forall u \in S .
$$

Consequently, we have that there exists a positive constant $A_{4}$ such that

$$
\begin{aligned}
I_{\lambda}(u) \leq & A_{1}\left(\|u\|_{\vec{p}(\cdot)}^{P_{+}^{+}}+\|u\|_{\vec{p}(\cdot)}^{P_{-}^{-}}\right)+A_{2}(\lambda)\left(\|u\|_{\vec{p}(\cdot)}^{m^{+}}+\|u\|_{\vec{p}(\cdot)}^{m^{-}}\right) \\
& +A_{3}-A_{4}\|u\|_{\frac{q^{-}(\cdot)}{q^{-}}}, \quad \forall u \in S .
\end{aligned}
$$

Hence

$$
\begin{aligned}
& A_{1}\left(\|u\|_{\vec{p}(\cdot)}^{P_{+}^{+}}+\|u\|_{\vec{p}(\cdot)}^{P_{-}^{-}}\right)+A_{2}(\lambda)\left(\|u\|_{\vec{p}(\cdot)}^{m^{+}}+\|u\|_{\vec{p}(\cdot)}^{m^{-}}\right) \\
& +A_{3}-A_{4}\|u\|_{\vec{p}(\cdot)}^{q^{-}} \geq 0, \quad \forall u \in M
\end{aligned}
$$

and since $m^{+}=P_{+}^{+}<q^{-}$, we conclude that $M$ is bounded in $W_{0}^{1, \vec{p}(\cdot)}(\Omega)$. Thus, Lemma 3 is proved.

Proof of Theorem 2. It is clear that $I_{\lambda} \in C^{1}\left(W_{0}^{1, \vec{p}(\cdot)}(\Omega), \mathbb{R}\right)$ is even and $I_{\lambda}(0)=$ 0 . Lemma 1 implies that $I_{\lambda}$ satisfies the Palais-Smale condition. On the other hand, Lemmas 2 and 3 show that conditions (I1) and (I2) are satisfied. The Mountain Pass Theorem can be applied to the functional $I_{\lambda}$. Thus, $I_{\lambda}$ has an unbounded sequence of critical values and consequently problem (13) has 
infinitely many weak solutions in $W_{0}^{1, \vec{p}(\cdot)}(\Omega)$. The proof of Theorem 2 is complete.

\section{Proof of Theorem 3}

We will use once more the critical point theory in order to prove Theorem 3.

Let $\lambda>0$ be arbitrary but fixed. The energy functional corresponding to problem (14) is defined as $J_{\lambda}: W_{0}^{1, \vec{p}(\cdot)}(\Omega) \rightarrow \mathbb{R}$,

$$
J_{\lambda}(u)=\int_{\Omega} \sum_{i=1}^{N} \frac{|\partial u|^{p_{i}(x)}}{p_{i}(x)} d x-\lambda \int_{\Omega} \frac{|u|^{m(x)}}{m(x)} d x+\int_{\Omega} \frac{|u|^{q(x)}}{q(x)} d x .
$$

Standard arguments assure that $J_{\lambda}$ is well-defined on $W_{0}^{1, \vec{p}(\cdot)}(\Omega)$ and $J_{\lambda} \in$ $C^{1}\left(W_{0}^{1, \vec{p}(\cdot)}(\Omega), \mathbb{R}\right)$ with the Fréchet derivative given by

$$
\begin{aligned}
\left\langle J_{\lambda}^{\prime}(u), v\right\rangle= & \int_{\Omega} \sum_{i=1}^{N}\left|\partial_{x_{i}} u\right|^{p_{i}(x)-2} \cdot \partial_{x_{i}} u \cdot \partial_{x_{i}} v d x-\lambda \int_{\Omega}|u|^{m(x)-2} u v d x \\
& +\int_{\Omega}|u|^{q(x)-2} u v d x
\end{aligned}
$$

for all $u, v \in W_{0}^{1, \vec{p}(\cdot)}(\Omega)$. Clearly, the weak solutions of problem (14) are exactly the critical points of functional $J_{\lambda}$.

Our goal is to show that $J_{\lambda}$ possesses a nontrivial global minimum point in $W_{0}^{1, \vec{p}(\cdot)}(\Omega)$. We start by establishing the following auxiliary result:

Lemma 4. The energy functional $J_{\lambda}$ is coercive on $W_{0}^{1, \vec{p}(\cdot)}(\Omega)$.

Proof. We recall that in [8, Lemma 4] it was proved that for any $a, b>0$ and $0<k<l$ the following inequality holds true

$$
a \cdot t^{k}-b \cdot t^{l} \leq a \cdot\left(\frac{a}{b}\right)^{\frac{k}{l-k}} \text { for all } t \geq 0 .
$$

Using relation (35), we infer that for any $x \in \Omega$ and $u \in W_{0}^{1, \vec{p}(\cdot)}(\Omega)$ we have

$$
\begin{aligned}
\frac{\lambda}{m^{-}}|u(x)|^{m(x)}-\frac{1}{q^{+}}|u(x)|^{q(x)} & \leq \frac{\lambda}{m^{-}}\left[\frac{\lambda q^{+}}{m^{-}}\right]^{\frac{m(x)}{q(x)-m(x)}} \\
& \leq \frac{\lambda}{m^{-}}\left[\left(\frac{\lambda q^{+}}{m^{-}}\right)^{\frac{m^{+}}{q^{-}-m^{+}}}+\left(\frac{\lambda q^{+}}{m^{-}}\right)^{\frac{m^{-}}{q^{+}-m^{-}}}\right]:=\mathfrak{C},
\end{aligned}
$$

where $\mathfrak{C}$ is a positive constant independent of $u$ and $x$.

Integrating the above inequality over $\Omega$, we get

$$
\frac{\lambda}{m^{-}} \int_{\Omega}|u|^{m(x)} d x-\frac{1}{q^{+}} \int_{\Omega}|u|^{q(x)} d x \leq \mathfrak{D},
$$

where $\mathfrak{D}$ is a positive constant independent of $u$. 
Next, we focus our attention on the elements $u \in W_{0}^{1, \vec{p}(\cdot)}(\Omega)$ with $\|u\|_{\vec{p}(\cdot)}$ $>1$.

For each $n$ and $i \in\{1, \ldots, N\}$ we define

$$
\xi_{n, i}= \begin{cases}P_{+}^{+}, & \text {if }\left|\partial_{x_{i}} u_{n}\right|_{p_{i}(\cdot)}<1 \\ P_{-}^{-}, & \text {if }\left|\partial_{x_{i}} u_{n}\right|_{p_{i}(\cdot)}>1\end{cases}
$$

By inequality (36), we get

$$
\begin{aligned}
J_{\lambda}(u) \geq & \frac{1}{P_{+}^{+}} \sum_{i=1}^{N} \int_{\Omega}\left|\partial_{x_{i}} u\right|^{p_{i}(x)} d x-\frac{\lambda}{m^{-}} \int_{\Omega}|u|^{m(x)} d x+\frac{1}{q^{+}} \int_{\Omega}|u|^{q(x)} d x \\
\geq & \frac{1}{P_{+}^{+}} \sum_{i=1}^{N}\left|\partial_{x_{i}} u\right|_{p_{i}(\cdot)}^{\xi_{n, i}}-\frac{\lambda}{m^{-}} \int_{\Omega}|u|^{m(x)} d x+\frac{1}{q^{+}} \int_{\Omega}|u|^{q(x)} d x \\
\geq & \frac{1}{P_{+}^{+}} \sum_{i=1}^{N}\left|\partial_{x_{i}} u\right|_{p_{i}(\cdot)}^{P_{-}^{-}}-\frac{1}{P_{+}^{+}} \sum_{\left\{i: \xi_{n, i}=P_{+}^{+}\right\}}\left(\left|\partial_{x_{i}} u\right|_{p_{i}(\cdot)}^{P_{-}^{-}}-\left|\partial_{x_{i}} u\right|_{p_{i}(\cdot)}^{P_{+}^{+}}\right) \\
& -\left(\frac{\lambda}{m^{-}} \int_{\Omega}|u|^{m(x)} d x-\frac{1}{q^{+}} \int_{\Omega}|u|^{q(x)} d x\right) \\
\geq & \frac{\|u\|_{\vec{p}(\cdot)}^{P_{-}^{-}}}{P_{+}^{+} N^{P_{-}^{-}-1}}-\frac{N}{P_{+}^{+}}-\mathfrak{D} .
\end{aligned}
$$

Thus, $J_{\lambda}(u) \geq \frac{\|u\|_{\vec{p}(\cdot)}^{P_{-}^{-}}}{P_{+}^{+} N^{P_{-}^{-}-1}}-\frac{N}{P_{+}^{+}}-\mathfrak{D}$ for all $u \in W_{0}^{1, \vec{p}(\cdot)}(\Omega)$ with $\|u\|_{\vec{p}(\cdot)}>1$. We infer that $J_{\lambda}(u) \rightarrow \infty$ as $\|u\|_{\vec{p}(\cdot)} \rightarrow \infty$. In other words, $J_{\lambda}$ is coercive in $W_{0}^{1, \vec{p}(\cdot)}(\Omega)$, completing the proof.

Proof of Theorem 3. By Lemma 4 we have that $J_{\lambda}$ is coercive. Moreover, a similar argument as the one used in the proof of [12, Lemma 3.4] shows that $J_{\lambda}$ is also weakly lower semi-continuous in $W_{0}^{1, \vec{p}(\cdot)}(\Omega)$. These facts enable us to apply [19, Theorem 1.2] in order to find that there exists $u_{\lambda} \in W_{0}^{1, \vec{p}(\cdot)}(\Omega)$ a global minimizer of $J_{\lambda}$ and thus, a weak solution of problem (14).

Next, we prove that $u_{\lambda}$ is not trivial for $\lambda$ large enough. Indeed, letting $t_{0}>1$ be a fixed real and choosing $\Omega_{1}$ an open subset of $\Omega$ with $\left|\Omega_{1}\right|>0$, we deduce that there exists $v_{0} \in C_{0}^{\infty}(\Omega) \subset W_{0}^{1, \vec{p}(\cdot)}(\Omega)$ such that $v_{0}(x)=t_{0}$ for any $x \in \overline{\Omega_{1}}$ and $0 \leq v_{0}(x) \leq t_{0}$ for any $x \in \Omega \backslash \overline{\Omega_{1}}$. Thus, we have

$$
\begin{aligned}
J_{\lambda}\left(v_{0}\right) & =\int_{\Omega} \sum_{i=1}^{N} \frac{\left|\partial_{x_{i}} v_{0}\right|^{p_{i}(x)}}{p_{i}(x)} d x-\lambda \int_{\Omega} \frac{\left|v_{0}\right|^{m(x)}}{m(x)} d x+\int_{\Omega} \frac{\left|v_{0}\right|^{q(x)}}{q(x)} d x \\
& \leq C-\frac{\lambda}{m^{+}} \int_{\Omega_{1}}\left|v_{0}\right|^{m(x)} d x
\end{aligned}
$$




$$
\leq C-\frac{\lambda}{m^{+}} t_{0}^{m^{-}} \cdot\left|\Omega_{1}\right|
$$

where $C$ is a positive constant.

Therefore, there exists $\lambda^{\star}>0$ such that $J_{\lambda}\left(v_{0}\right)<0$ for any $\lambda \geq \lambda^{\star}$. It follows that $J_{\lambda}\left(u_{\lambda}\right)<0$ for any $\lambda \geq \lambda^{\star}$ and thus, we find that $u_{\lambda}$ is a nontrivial weak solution of problem (14) for $\lambda$ large enough. This completes the proof of Theorem 3.

Acknowledgments. The authors were partially supported by the grant CNCSIS-UEFISCSU PN-II-ID-PCE-2011-3-0075, Analysis, Control and Numerical Approximations of Partial Differential Equations.

\section{References}

[1] D. E. Edmunds, J. Lang, and A. Nekvinda, On $L^{p(x)}$ norms, Proc. R. Soc. Lond. Ser. A Math. Phys. Eng. Sci. 455 (1999), no. 1981, 219-225.

[2] D. E. Edmunds and J. Rákosník, Density of smooth functions in $W^{k, p(x)}(\Omega)$, Proc. Roy. Soc. London Ser. A 437 (1992), no. 1899, 229-236.

[3] _ Sobolev embedding with variable exponent, Studia Math. 143 (2000), no. 3, $267-293$.

[4] X. Fan, J. Shen, and D. Zhao, Sobolev embedding theorems for spaces $W^{k, p(x)}(\Omega)$, J. Math. Anal. Appl. 262 (2001), no. 2, 749-760.

[5] X. L. Fan and D. Zhao, On the Spaces $L^{p(x)}(\Omega)$ and $W^{m, p(x)}(\Omega)$, J. Math. Anal. Appl. 263 (2001), no. 2, 424-446.

[6] I. Fragalà, F. Gazzola, and B. Kawohl, Existence and nonexistence results for anisotropic quasilinear elliptic equations, Ann. Inst. H. Poincaré Anal. Non Linéaire 21 (2004), no. $5,751-734$.

[7] O. Kováčik and J. Rákosník, On spaces $L^{p(x)}$ and $W^{1, p(x)}$, Czechoslovak Math. J. 41(116) (1991), no. 4, 592-618.

[8] M. Mihăilescu, On a class of nonlinear problems involving a $p(x)$-Laplace type operator, Czechoslovak Math. J. 58(133) (2008), no. 1, 155-172.

[9] M. Mihăilescu and G. Moroşanu, Existence and multiplicity of solutions for an anisotropic elliptic problem involving variable exponent growth conditions, Appl. Anal. 89 (2010), no. 2, 257-271.

[10] _ On an eigenvalue problem for an anisotropic elliptic equation involving variable exponents, Glasg. Math. J. 52 (2010), no. 3, 517-527.

[11] M. Mihăilescu, P. Pucci, and V. Rădulescu, Eigenvalue problems for anisotropic quasilinear elliptic equations with variable exponent, J. Math. Anal. Appl. 340 (2008), no. 1, 687-698.

[12] M. Mihăilescu and V. Rădulescu, A multiplicity result for a nonlinear degenerate problem arising in the theory of electrorheological fluids, Proc. R. Soc. Lond. Ser. A Math. Phys. Eng. Sci. 462 (2006), no. 2073, 2625-2641.

[13] J. Musielak, Orlicz Spaces and Modular Spaces, Lecture Notes in Mathematics, Vol. 1034, Springer, Berlin, 1983.

[14] S. M. Nikol'skii, On imbedding, continuation and approximation theorems for differentiable functions of several variables, Russian Math. Surveys 16 (1961), 55-104.

[15] P. Rabinowitz, Minimax methods in critical point theory with applications to differential equations, Expository Lectures from the CBMS Regional Conference held at the University of Miami, American Mathematical Society, Providence, RI. 1984.

[16] J. Rákosník, Some remarks to anisotropic Sobolev spaces. I, Beiträge Anal. 13 (1979), $55-68$. 
[17] _ Some remarks to anisotropic Sobolev spaces. II, Beiträge Anal. 15 (1981), 127140 .

[18] M. Ruzicka, Electrorheological Fluids Modeling and Mathematical Theory, SpringerVerlag, Berlin, 2002

[19] M. Struwe, Variational Methods: Applications to Nonlinear Partial Differential Equations and Hamiltonian Systems, Springer, Heidelberg, 1996

[20] M. Troisi, Teoremi di inclusione per spazi di Sobolev non isotropi, Ric. Mat. 18 (1969), $3-24$.

[21] L. Ven'-tuan, On embedding theorems for spaces of functions with partial derivatives of various degree of summability, Vestnik Leningrad. Univ. 16 (1961), 23-37.

Mihai Mihăilescu

Department of Mathematics

UNIVERSITY OF CRAIOVA

200585 Craiova, Romania

AND

InSTITUTE OF MATHEMATICS

Simion Stollow of the Romanian Academy

010702 Bucharest, Romania

E-mail address: mmihailes@yahoo.com

Denisa Stancu-Dumitru

Department of Mathematics

University OF CRAIOVA

200585 Craiova, Romania

AND

Institute of Mathematics

Simion Stollow of the Romanian Academy

010702 Bucharest, Romania

E-mail address: denisa.stancu@yahoo.com 